\title{
A Systematic Review and Meta-analysis of the Role of Toll-like Receptor 9 in Alzheimer's Disease: The Protocol for a Systematic Review
}

\author{
Nasrin Hosseini ${ }^{1,}{ }^{*}$, Shabnam Nadjafi ${ }^{1}$, Leila Janani $\mathbb{i}^{2}{ }^{2}$, Zahra Faraji ${ }^{3}$, Behnaz Ashtari ${ }^{4}$, Seyed \\ Behnamedin Jameie ${ }^{1}$, Mona Farhadi ${ }^{5}$ and Batool Okhovat Isfahani ${ }^{6}$ \\ ${ }^{1}$ Neuroscience Research Center, Iran University of Medical Sciences, Tehran, Iran \\ ${ }^{2}$ Department of Biostatistics, School of Public Health, Iran University of Medical Sciences, Tehran, Iran \\ ${ }^{3}$ Central Library, Iran university of Medical Sciences, Tehran, Iran \\ ${ }^{4}$ Department of Medical Nanotechnology, Faculty of Advanced Technology in Medicine, Iran University of Medical Sciences, Tehran, Iran \\ ${ }^{5}$ Department of Microbiology, Karaj Branch, Islamic Azad University, Karaj, Iran \\ ${ }^{6}$ Department of Epidemiology and Biostatistics, School of Public Health, Tehran University of Medical Sciences, Tehran, Iran \\ "Corresponding author: Neuroscience Research Center, Iran University of Medical Sciences, Tehran, Iran. Email: hosseini.n58@gmail.com
}

Received 2021 March 08; Revised 2021 March 19; Accepted 2021 April 01.

\begin{abstract}
Context: Alzheimer's disease (AD) is a neurodegenerative disease affecting many people around the world. Recently, it has been reported that toll-like receptors (TLRs) play a role in AD; therefore, the present study aimed to systematically review the studies and to meta-analyze the role of toll-like receptor 9 (TLR9) in AD.

Methods: Seven main electronic databases, including PubMed/MEDLINE, Web of Science, Embase, Scopus, CINAHL, Cochrane, and Google Scholar, will be considered with no language restrictions. Full texts of articles will be prepared by a determined search strategy. Studies including the assessment of TLR9 function in adults with AD, published before June 152020 , will be considered. Hence, this protocol will be presented based on the preferred reporting items for systematic reviews and meta-analyses (PRISMA) statements for protocols. The related results and data analyses will be provided in the final review. This paper plans the protocol for a systematic review identifying TLR9 up-regulation and down-regulation in adults with AD.

Conclusions: The meta-analysis of TLR9 may subsequently provide attractive therapeutic tools for AD.
\end{abstract}

Keywords: Alzheimer's Disease, Toll-like Receptors, Therapy, Systematic Review

\section{Context}

Alzheimer's disease (AD) is known as a neurodegenerative disease associated with aging. The clinical characterization of $\mathrm{AD}$ is presented through developing cognitive function deterioration in adult life. Definite forms of memory and language losses are the typical initial symptoms of $A D$ (1). According to histopathological aspects of $A D$, dense protein aggregation containing extracellular $\mathrm{A} \beta$ (amyloid beta) plaques and intracellular neurofibrillary tangles are the known neuropathological characteristics in AD brain $(2,3)$. Also, tau protein plays a key role in $A \beta$ toxicity; thus, tau protein levels in the brain are significantly correlated with cognitive impairments (2). Both intracellular and extracellular accumulations of $\mathrm{A} \beta$ peptide participate in the primary event. They stem from the impairment of clearance pathways and this can aggregate to form insoluble plaques (2). The immunological approach has proved the activation of microglia in AD tissue, particularly in surrounding amyloid plaques (4).
In recent decades, the involvement of toll-like receptors (TLRs) in AD has been increasingly suggested (4). TLRs are a class of pattern recognition receptors (PRRs) in mammals (5). Briefly, germline-encoded PRRs are employed by the innate immune system for the initial detection of microbes (5). However, PRRs may determine specific molecular patterns, including pathogen-associated molecular patterns (PAMPs) and damage-associated molecular patterns (DAMPs) (5). In brief, PAMPs are microbe-specific molecular signatures; however, DAMPs are self-derived molecules derived from damaged cells (5). In fact, a variety of molecular components originated from microorganisms and classified into lipid, protein, and nucleic acids have been known as most of the TLR ligands (6). In humans and mice, TLRs belong to a family with 10 (TLR1-TLR10) and 12 (TLR1-TLR9, TLR11-TLR13) members, respectively. TLRs are found in cell surfaces and some intracellular organelles, including the endoplasmic reticulum (ER), endosome, lysosome, or endolysosome (5). 
Structurally, the TLR is a trans-membrane protein with two binding sites for ligands and receptors. The inflammatory process and other signaling and responses related to the adaptive immune system can also be triggered as specific ligands recognized by TLRs (4). Currently, it is not clear yet that if the role of TLRs in AD may be beneficial or contribute to AD progression (7)). TLR9 as an intracellular receptor can recognize viral double-stranded RNA and unmethylated cytosine-guanosine (CpG) islands in DNA (8, 9). There is evidence that the stimulation of TLR9 by CPG oligodeoxynucleotides (ODNs) leads to a dramatic reduction of amyloid load in a mouse model of AD (9). Likewise, there are reports showing the relation of TLR9 to AD in humans $(10,11)$. Accordingly, in this systematic review, we intend to evaluate the changes of TLR9 in AD patients.

\section{Methods}

The conducted review will be presented based on the PRISMA statement (12). The PRISMA flowchart will be used to explain the number of initial studies (included and deleted) at the various stages of this systematic review (Appendix 2 in Supplementary File).

\subsection{Patient and Public Involvement}

This is a protocol for previously published articles; thus, patients or public and related data are not necessary to be assessed or included in any section of this protocol development.

\subsection{Criteria}

All articles related to TLR9 expression, AD dementia, mild cognitive impairment (MCI), and subjective cognitive decline (SCD), which were available and published on mentioned searched databases before June 15 2020, will be collected. The inclusion and exclusion criteria are provided in the following sections.

\subsection{Population}

Adult patients with dementia due to $\mathrm{AD}$ will be included. Other cognitive disorders due to alcohol consumption or drug abuse, central nervous system (CNS) injuries like subdural hematoma, tumor, or infection, as well as other neurological complications such as Huntington's disease or Parkinson's disease, will be excluded.

\subsection{Comparator}

The control group will consist of healthy old adults. Those with cognitive impairments, neurocognitive complications, and disrupted daily functions will be excluded.

\subsection{Outcomes}

We will only consider quantitative measurements.

\subsection{Study Type}

The observational studies, including cross-sectional or case-control and cohort studies that investigated TLR9 and $\mathrm{AD}$ in human studies, will be included.

There will be no language restrictions during the papers' searching. All papers should be studies published in peer-reviewed journals.

\subsection{Information Sources}

The following databases will be searched for relevant studies, published between January 1, 1990, and June 15, 2020, with no language restrictions: PubMed/MEDLINE, Web of Science, Embase, Scopus, CINAHL, Cochrane, and Google Scholar. The search strategy syntax will be altered to be used in the mentioned databases. In final analyses, the searches will be performed again, and any new study will be considered for inclusion. Also, case reports, reviews, editorials, letters, and case series articles will be removed. To do so, a search utilizing the keywords "Alzheimer's disease" and "toll-like receptor9" will be performed. An example of a conducted PubMed search strategy is provided in Appendix 3 in Supplementary File. It should be noted that search syntax will be adjusted in accordance with the used databases. The search terms will be found both in EMBASE (EMTREE) and in MEDLINE/PubMed (Medical Subject Headings/MeSH), and a combination of these terms will be used to produce an appropriate electronic search strategy. The titles, abstracts, and keywords of the studies will be considered. Grey literature will be collected using Google Scholar, Open Grey, ClinicalTrials.gov, and the International Clinical Trials Registry Platform. Unpublished studies, including conference papers, scientific meetings, or theses, will be included in the search to mitigate publication bias. We will also contact the authors of these eligible conference papers through emails, and we will ask them for full texts, if necessary.

\subsection{Search Strategy}

To make the search strategy highly sensitive, in final analyses, the searches will be performed again, and any new study will be considered for inclusion. The searches will be performed in titles, abstracts, and full-texts. A dual independent review will be carried out to the search strategy. This will also be used to minimize random errors and bias for the studies' identification and assessment processes. The independent librarian will recheck the search strategy. 


\subsection{Data Management}

Data management will be conducted using the EndNote software version X7. One of the researchers (Z.F.) will import the search results for each database into the EndNote library, will remove the duplicate records of each database into the EndNote library, and then will remove the duplicate records.

\subsection{Study Selection}

The three-step model will be used for the inclusion of proper studies in the present review. The first step will be to examine the relevance of the titles and abstracts of the selected papers. Articles without abstracts or incomplete abstracts will be considered for full-text analysis. In the next step, studies with eligibility for our study will be checked by two researchers (N.H. and S.N.) separately. In the final step, full texts will be assessed by reviewers to reveal their relevance. To resolve any existing disagreement, the third reviewer (F.K.), who is experienced in this field, will be consulted. The reasons for the exclusion of the studies will be recorded at each stage. The researcher (Z.F.) will perform the search, review, and initial selection of articles based on the search strategy and in accordance with the PRISMA-P statement simultaneously (13), and will save the results of each database, separately. For each database, duplicates will be determined, and newly found cases will also be chosen and saved. Then, two researchers (S.N. and N.H.) will review the final list of references for all selected articles and grey literature to individualize other relevant articles.

\subsection{Data Extraction}

A form will be prepared for data extraction, and two reviewers (N.H. and S.N.) will evaluate and extract data independently.

The data extraction from the screened papers will include the following:

1) Study characteristics such as paper ID, publication date, the first author's name, country, publication language, setting locations, study design, sample selection criteria, sample size, diagnostic criteria, and measured outcomes.

2) General characteristics of participants, including gender, age, and ethnicity: The missing data will be asked from the corresponding author through email. The included studies will be categorized based on the outcome data and the clinical examination by which the participants are diagnosed. If there is any disagreement on the inclusion of a study, the third reviewer will resolve it. Also, all reasons for the exclusion of a study will be explained in a documented table.
If the data presented in the research report are incomplete, the researchers will contact the corresponding author for more information to manage the data in specific circumstances, determined by the Cochrane Institute. If the authors do not respond to the first email, we will send up to three reminder emails. After sending three reminder emails, if we do not receive any answer, the reviewers will consider the incomplete information as missing data.

\subsection{Quality Assessment}

The included papers will be analyzed for reliability or internal validity to specify the risk of bias, including selection bias, performance bias, detection bias, attrition bias, analysis bias, and reporting bias.

Two reviewers (S.N. and N.H.) will evaluate the methodological quality of primary studies independently by the Joanna Briggs Institute (JBI; 2018) Critical Appraisal Checklist for Analytical Cross-Sectional studies. Also, case-control and cohort studies will be assessed by other developed tools; if necessary, this will be implemented for all included studies to evaluate the study quality. In case of uncertainty or disagreement between the reviewers, an independent reviewer (L.J.) will be conferred through dialogue to gain consensus. The publication bias will be evaluated by funnel plots (study results against accuracy plots) and Begg's and Egger's tests.

\subsection{Meta-analytic Approach}

Heterogeneity will be assessed to detect the scale variation for effect estimate caused by heterogeneity instead of chance. The heterogeneity in primary studies will be evaluated by $\chi^{2}$ test (at the level of $10 \%$ will be considered as significant) and $\mathrm{I}^{2}$ statistic (50\% - 90\% will show significant heterogeneity). The synthesized effect size will be presented in the form of the standard mean differences with 95\% CI. If the model of heterogeneity provides meta-analysis permission, a random-effects model will be used. Therefore, meta-analysis will be structured using the Comprehensive Meta-Analysis (CMA V.3, Biostat, Englewood, USA). The standard mean differences represent the differences between the means of two groups divided by the standard deviation (SD). For the meta-analysis, the data will be presented as continuous outcomes and the random-effects model will be used for the variations of exciting methodology between studies. Moreover, the reason for high levels of heterogeneity will be determined. If we succeed in finding the sources of heterogeneity, we will use subgroup analysis to present pooled results in the relevant subgroups. The results will be considered as significant for p-values $<0.05$. 


\section{Discussion}

The main aim of the present study is to systematically review TLR9, having been noticed in studies with participants diagnosed with AD. The results of this review will add information regarding TLR9 up-regulation and downregulation in older adults with $\mathrm{AD}$. This will help design potential therapeutic programs for individuals at risk for AD. We suggest that this review will help many stakeholders who are concerned about treating AD.

\subsection{Dissemination Plans}

This systematic review will be submitted to a peerreviewed journal both local and international scientific meetings.

\subsection{Strengths and Limitations of This Study}

The present systematic review evaluates the role of TLR9 in AD. The method is based on a comprehensive search strategy containing studies in any language and covering the period from January 1, 1990, to June 15, 2020.

The review protocol is prepared in line with the protocol guidelines of systematic reviews and meta-analyses (Appendix 1 in Supplementary File), and the articles' quality will be evaluated by using validated tools.

Comparison between studies may not be possible due to heterogeneity in measurement tools.

\section{Supplementary Material}

Supplementary material(s) is available here [To read supplementary materials, please refer to the journal website and open PDF/HTML].

\section{Footnotes}

Authors' Contribution: Study concept and design: $\mathrm{NH}$, SN, and LJ. Search strategies: NH, ZF, and SN. Analysis and interpretation of data, screening potential studies, extracting data, and assessing quality: $\mathrm{NH}, \mathrm{SN}$, and LJ. Reviewing the work: SBJ, BA, BOI, and MF. Initial drafting and critical revision of the manuscript: $\mathrm{NH}$ and $\mathrm{SN}$. Also all authors give feedback to the final draft of the protocol; approving the manuscript for submission: NH. All authors read and approve the final manuscript.

Conflict of Interests: There is no conflict of interests.

Ethical Approval: The ethical approval of this study was obtained from Iran University of Medical Science (no.: IR.IUMS.REC 1396.32121).
Funding/Support: This systematic review is funded by a grant from Iran University of Medical Sciences.

\section{References}

1. Auld DS, Kornecook TJ, Bastianetto S, Quirion R. Alzheimer's disease and the basal forebrain cholinergic system: relations to beta-amyloid peptides, cognition, and treatment strategies. Prog Neurobiol. 2002;68(3):209-45. doi: 10.1016/s0301-0082(02)00079-5. [PubMed: 12450488].

2. Jevtic S, Sengar AS, Salter MW, McLaurin J. The role of the immune system in Alzheimer disease: Etiology and treatment. Ageing Res Rev. 2017;40:84-94. doi: 10.1016/j.arr.2017.08.005. [PubMed: 28941639].

3. Landreth GE, Reed-Geaghan EG. Toll-like receptors in Alzheimer's disease. Curr Top Microbiol Immunol. 2009;336:137-53. doi: 10.1007/978-3642-00549-7_8. [PubMed:19688332]. [PubMed Central: PMC3032986].

4. Su F, Bai F, Zhou H, Zhang Z. Microglial toll-like receptors and Alzheimer's disease. Brain Behav Immun. 2016;52:187-98. doi: 10.1016/j.bbi.2015.10.010. [PubMed: 26526648].

5. Kawasaki T, Kawai T. Toll-like receptor signaling pathways. Front Immunol. 2014;5:461. doi: 10.3389/fimmu.2014.00461. [PubMed: 25309543]. [PubMed Central: PMC4174766].

6. Kaisho T, Akira S. Toll-like receptor function and signaling. J Allergy Clin Immunol. 2006;117(5):979-87. quiz 988. doi: 10.1016/j.jaci.2006.02.023. [PubMed: 16675322].

7. Amor S, Puentes F, Baker D, van der Valk P. Inflammation in neurodegenerative diseases. Immunology. 2010;129(2):154-69. doi: 10.1111/j.1365-2567.2009.03225.x. [PubMed: 20561356]. [PubMed Central: PMC2814458].

8. Le Page A, Bourgade K, Lamoureux J, Frost E, Pawelec G, Larbi A, et al. NK Cells are Activated in Amnestic Mild Cognitive Impairment but not in Mild Alzheimer's Disease Patients. J Alzheimers Dis. 2015;46(1):93-107. doi: 10.3233/JAD-143054. [PubMed: 25720398].

9. Scholtzova H, Kascsak RJ, Bates KA, Boutajangout A, Kerr DJ, Meeker $\mathrm{HC}$, et al. Induction of toll-like receptor 9 signaling as a method for ameliorating Alzheimer's disease-related pathology. J Neurosci. 2009;29(6):1846-54. doi: 10.1523/JNEUROSCI.5715-08.2009. [PubMed: 19211891]. [PubMed Central: PMC2699573].

10. Fiala M, Liu PT, Espinosa-Jeffrey A, Rosenthal MJ, Bernard G, Ringman JM, et al. Innate immunity and transcription of MGAT-III and Tolllike receptors in Alzheimer's disease patients are improved by bisdemethoxycurcumin. Proc Natl Acad Sci U S A. 2007;104(31):12849-54. doi: 10.1073/pnas.0701267104. [PubMed: 17652175]. [PubMed Central: PMC1937555].

11. Wang YL, Tan MS, Yu JT, Zhang W, Hu N, Wang HF, et al. Toll-like receptor 9 promoter polymorphism is associated with decreased risk of Alzheimer's disease in Han Chinese. J Neuroinflammation. 2013;10:101. doi: 10.1186/1742-2094-10-101. [PubMed: 23957925]. [PubMed Central: PMC3765501].

12. Moher D, Liberati A, Tetzlaff J, Altman DG, Prisma Group. Preferred reporting items for systematic reviews and meta-analyses: the PRISMA statement. Ann Intern Med.2009;151(4):264-9. W64. doi:10.7326/00034819-151-4-200908180-00135. [PubMed: 19622511].

13. Shamseer L, Moher D, Clarke M, Ghersi D, Liberati A, Petticrew M, et al. Preferred reporting items for systematic review and metaanalysis protocols (PRISMA-P) 2015: elaboration and explanation. BMJ 2015;350:g7647. doi: 10.1136/bmj.g7647. [PubMed: 25555855]. 